\title{
Appendiceal adenocarcinoma found by surgery for acute appendicitis is associated with older age
}

John P. Skendelas ${ }^{1,2,3,4 \dagger}$, Victor S. Alemany ${ }^{1 \dagger}$, Vincent Au ${ }^{1,3}$, Devika Rao ${ }^{1,2,3,4}$, John McNelis ${ }^{1,2,4}$ and Peter K. Kim ${ }^{1,2,4,5^{*}}$

\begin{abstract}
Background: Appendectomy for acute appendicitis is the most common procedure performed emergently by general surgeons in the United States. The current management of acute appendicitis is increasingly controversial as non-operative management gains favor. Although rare, appendiceal neoplasms are often found as an incidental finding in the setting of appendectomy. Criteria and screening for appendiceal neoplasms are not standardized among surgical societies.
\end{abstract}

Methods: The National Surgical Quality Improvement Program (NSQIP) database was queried for all patients who underwent appendectomy over a 9-year period (2010-2018). Over the same time period, patients who underwent appendectomy in two municipal hospitals in The Bronx, New York City, USA were reviewed.

Results: We found a $1.7 \%$ incidence of appendiceal neoplasms locally and a $0.53 \%$ incidence of appendiceal tumors in a national population sample. Both groups demonstrated an increased incidence of appendiceal carcinoma by age. This finding was most pronounced after the age of 40 in both local and national populations. In our study, the incidence of appendiceal tumors increased with each decade interval up to the age of 80 and peaked at $2.1 \%$ in patients between 70 and 79 years.

Conclusions: Appendiceal adenocarcinomas were identified in patients with acute appendicitis that seem to be associated with increasing age. The presence of an appendiceal malignancy should be considered in the management of older patients with acute appendicitis before a decision to embark on non-operative therapy.

Keywords: Acute appendicitis, Adenocarcinoma, Neuroendocrine tumor, Mucinous neoplasm, Emergency general surgery, Acute care surgery

\section{Background}

The incidence of appendicitis in the United States has been reported between 82 and 111 per 100,000 population per year, with a life-time risk of 1 in 15 (6.7\%) [1].

\footnotetext{
*Correspondence: kim.peter.p01@gmail.com

${ }^{\dagger}$ John P. Skendelas and Victor S. Alemany are co-first authors of this manuscript

5 Jacobi Medical Center, 1400 Pelham Parkway, Building 1, Room 510, Bronx, NY 10461, USA

Full list of author information is available at the end of the article
}

Appendectomy for acute appendicitis is the most common emergency intra-abdominal operation performed by general surgeons, and approximately 300,000 appendectomies are performed annually in the USA alone [2]. In recent years, there has been increased interest in nonoperative management of acute appendicitis as safe and feasible first-line therapy [3], similar to the management approach in other countries [4-7]. Historically, antibiotic therapy has been used in $40-45 \%$ of patients in Europe compared to fewer than $5 \%$ in the United States $[8,9]$; 
however, this approach has been challenged due to the ongoing "Comparison of Drugs versus Appendectomy" (CODA) trial, which found that early outcomes of medical management of appendicitis, with or without appendicolith, was non-inferior to surgery [10].

The COVID-19 pandemic has had extensive effects across the healthcare spectrum, including the management of acute appendicitis. Conservative management of mild appendicitis was practiced in several centers across the globe during the pandemic [11]. Non-operative management during the COVID-19 pandemic did not increase complications in one center [12], although several questions remain regarding long-term outcomes in these patients. Unintended consequences of nonoperative management may include increased incidence of antibiotic-resistant organisms and alterations to the microbiome [3]. Nevertheless, this approach has been adopted by the American College of Surgeons during the current pandemic and will likely continue [13].

This shift in clinical practice has raised concerns regarding the risk of missing an appendiceal neoplasms in patients who receive non-operative therapy [14]. In the CODA trial, neoplasms were identified in 9 participants $(0.6 \%)$, in spite of strict inclusion criteria to screen for underlying or likely malignancy [10]. Similarly, in the post-hoc analysis of the "Multicenter Study of the Treatment of Appendicitis in America: Acute, Perforated, and Gangrenous" (MUSTANG) trial [15, 16], neoplasms were associated with patient age greater than 40 and appendiceal size greater than $1 \mathrm{~cm}$ on cross-sectional imaging.

While the overall incidence remains low, there are neither established criteria to determine which patients are eligible for medical management nor criteria for patients who may require oncologic surveillance after non-operative treatment for appendicitis. The aims of this study were (1) to determine the incidence of appendiceal neoplasms from the National Surgical Quality Improvement Program (NSQIP) database and (2) compare these findings in two urban municipal hospitals in the Bronx, New York City.

\section{Methods}

The National Surgical Quality Improvement Program (NSQIP) personal use file database was queried using the ICD-9 codes from May 1, 2010 to December 31, 2018 for 'appendicitis": 540 (540.0, 540.1, 540.9), 541, 542; "appendectomy as an outpatient procedure": 44,950, 44,955, 44,960, 44,870, 44,979, 44,900, 44,901; "appendectomy as an inpatient procedure": 45.72, 45.73, 47, 47.01, 47.09, 47.1, 47.11, 47.19, 47.2, 47.92, 47.99; "neoplasm of the appendix": 153.9 and 152.9; and the ICD 10 codes for "appendicitis" K35, K36, "other appendicitis" K37 and K38; "neoplasm of the appendix", "neoplasm of the intestinal tract", "small intestine and colon unspecified" C17.9, C18.9, C26.0 and C18.1 and for "appendectomy": 0DTJ0ZZ, 0DTJ4ZZ, 0DTJ7ZZ, 0DTJ8ZZ. During this time period there were over 2.8 million patients in the database. Cases of appendiceal neoplasm were then categorized by age, type of procedure, and pathologic diagnosis. In the database there were only three pathologies of appendiceal neoplasm specified: adenocarcinoma, malignant carcinoid, and unspecified. Cases with known benign pathology after appendectomy were excluded from analysis.

Institutional data were retrospectively collected from the electronic medical records of all patients diagnosed with acute appendicitis in the period January, 2010 through December, 2018 in two Bronx municipal hospitals. Records were further confirmed with an institutional Tumor Registry and Pathology database to ensure inclusion of all malignant cases. All 1989 surgical pathology reports were reviewed to evaluate for neoplasms in appendectomy specimens. Data acquired included age at diagnosis, sex, prior colonoscopy records, radiographic findings, as well as final pathology and oncologic outcomes.

Continuous variables were expressed as medians with interquartile range (IQR). Categorical variables were described with number counts and percentages. Continuous variables were compared using Mann-Whitney $\mathrm{U}$ test. Categorical variables were compared using Chi-square tests. All statistical tests were two-tailed and p-value less than 0.05 was considered significant. All analyses were performed using SPSS Version 27.0 (IBM Corp, Armonk, NY, USA). All data collection, chart review, and methods were performed with approval by the hospital's Institutional Review Board (IRB) of the Albert Einstein College of Medicine (IRB Protocol \#20177530 ) and in accordance with all relevant guidelines and regulations.

\section{Results}

The National Surgical Quality Improvement Program (NSQIP) database was queried between 2010 and 2018. A total of 154,596 appendectomies performed in adult (age $\geq 18$ years) patients were identified, which included 812 cases of appendiceal cancer (for an incidence of $0.53 \%)$. The incidence of appendiceal tumors stratified by age are summarized in Fig. 1 . The combined incidence of appendiceal neoplasms identified in these data are $0.4 \%, 0.8 \%, 1.6 \%, 2.1 \%$, and $1.6 \%$ respectively for age $40-49,50-59,60-69,70-79$, and $>80$. For patients older than 40 years, $1 \%$ of appendectomy specimens demonstrated an appendiceal neoplasm. From the 812 appendiceal neoplasms, 681 adenocarcinomas of the appendix $(0.4 \%)$ were identified. For these patients, 290 underwent 


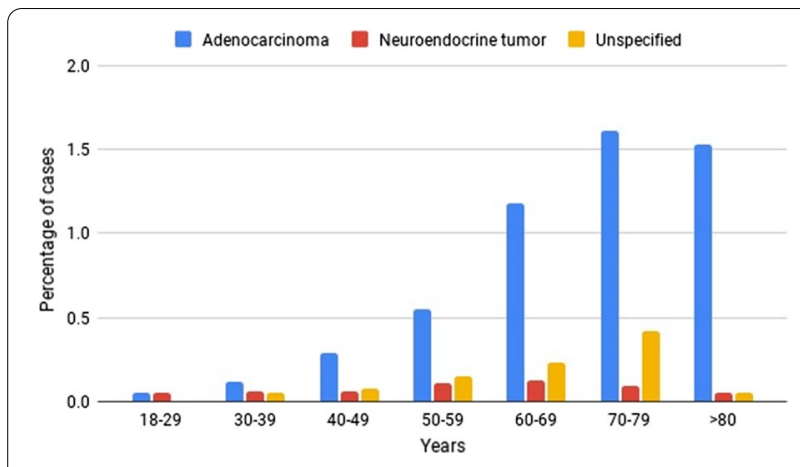

Fig. 1 The incidence of adenocarcinoma, neuroendocrine, and low grade appendiceal mucinous neoplasm (LAMN) obtained from the National Surgical Quality Improvement Program (NSQIP) database from 2010 to 2018 categorized into 10 year age groups

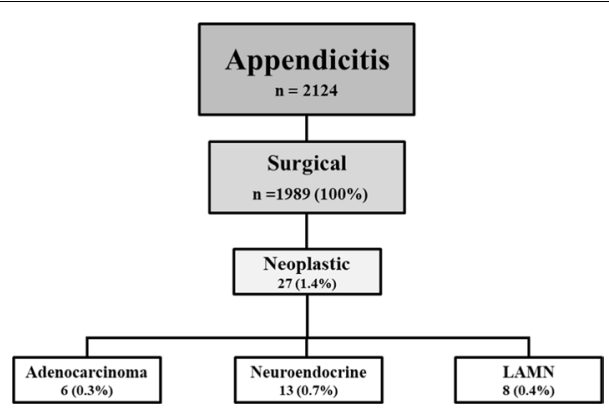

Fig. 2 The total number of local cases of appendicitis and those managed surgically reviewed from two Bronx municipal hospitals for the period 2010 through 2018. The number of cases found to be appendiceal cancers are further categorized into adenocarcinoma, neuroendocrine, and low grade appendiceal mucinous neoplasm (LAMN) appendectomy and 524 underwent colectomy; 133 patients underwent both procedures.

A total of 2124 medical charts from patients diagnosed with acute appendicitis were reviewed in two municipal hospitals using the electronic medical records from 2010 through 2018. At these institutions, 1989 patients (93.6\%) underwent operative treatment for acute appendicitis, with 1104 patients $(52.0 \%$ of total surgeries) undergoing laparoscopic appendectomy, 852 for open surgery (40.1\%) and $32(1.5 \%)$ who underwent conversion from laparoscopic to open approach. From the operative cohort, 27 patients were diagnosed with appendiceal neoplasm after the pathologic study of the removed appendix which reflected an incidence of $1.4 \%$ in this study population over 9 years (Fig. 2).

The median age of the neoplasm cohort was 49.0 years IQR [39.3-54.2]), with approximately $2: 1$, female to male ratio (70.4\% female). Remaining demographics and clinical factors are presented by non-adenocarcinoma (low-grade appendiceal mucinous neoplasm or LAMN, neuroendocrine) and appendiceal adenocarcinoma in Table 1 . Of note, 11 of 27 patients (40.7\%) presented with complicated disease (e.g. perforation, small bowel obstruction, or malignant ascites), whereas only 7 of 27 patients (25.9\%) had a prior colonoscopy. In addition, all neuroendocrine tumors were Grade 1 and categorized as benign.

The entire series of patients with appendiceal neoplasms are presented in Table 2 with associated age at diagnosis, appendiceal size on imaging, surgical approach, pathology, and stage. The final pathology revealed six cases of adenocarcinoma $(0.3 \%$ of appendectomy specimens), 13 neuroendocrine tumors $(0.7 \%)$,

Table 1 Demographics

\begin{tabular}{|c|c|c|c|}
\hline & Appendiceal adenocarcinoma $(n=6)$ & $\begin{array}{l}\text { Composite non-adenocarcinoma }{ }^{a} \\
(n=21)\end{array}$ & p-value \\
\hline Age-years [IQR] & $65.0[52.8-75.3]$ & $47.0[25.5-54.5]$ & 0.004 \\
\hline Female sex-no. (\%) & $5(83)$ & $14(67)$ & 0.633 \\
\hline Previous colonoscopy—no. (\%) & $2(33)$ & $5(24)$ & 0.633 \\
\hline Imaging size, median—cm [IQR] & $1.4[1.3-3.0]$ & $1.2[1.1-1.8]$ & 0.263 \\
\hline Emergency department presentation—no. (\%) & $6(100)$ & $18(86)$ & 0.810 \\
\hline Operative intervention—no. (\%) & & & 0.552 \\
\hline Open & $4(67)$ & $9(43)$ & \\
\hline Laparoscopic & $2(33)$ & $11(52)$ & \\
\hline Converted & 0 & $1(5)$ & \\
\hline Complicated-no. $(\%)^{\mathrm{b}}$ & $3(50)$ & $8(38)$ & 0.662 \\
\hline
\end{tabular}

$I Q R$ interquartile range

${ }^{\text {a }}$ Patients with appendectomy specimen pathology consistent with low-grade appendiceal mucinous neoplasm or neuroendocrine tumors

${ }^{b}$ Complicated cases included radiologic evidence of perforation $(n=7)$, small bowel obstruction $(n=3)$, and malignant ascites $(n=1)$ 
Table 2 Summary of appendiceal neoplasms

\begin{tabular}{|c|c|c|c|c|c|}
\hline Patient ID & $\begin{array}{l}\text { Age at } \\
\text { presentation } \\
\text { (years) }\end{array}$ & $\begin{array}{l}\text { Appendiceal } \\
\text { imaging size } \\
(\mathrm{cm})\end{array}$ & Pathology & Stage (TNM) & Surgical procedure \\
\hline 1 & 73 & 3.1 & Adenocarcinoma & $\begin{array}{l}\| \mathrm{A} \\
(\mathrm{T} 3, \mathrm{NO}, \mathrm{MO})\end{array}$ & Open right hemicolectomy \\
\hline 2 & 67 & 3.0 & Adenocarcinoma & IV & $\begin{array}{l}\text { Diagnostic laparoscopy with appendectomy, omental and peritoneal } \\
\text { biopsy }\end{array}$ \\
\hline 3 & 63 & 1.2 & Adenocarcinoma & $\begin{array}{l}\| \mathrm{A} \\
(\mathrm{T} 3, \mathrm{NO}, \mathrm{MO})\end{array}$ & Open right hemicolectomy \\
\hline 4 & 82 & 1.4 & Adenocarcinoma & $\begin{array}{l}\text { IV } \\
(\mathrm{T} 4, \mathrm{~N} 2, \mathrm{M} 1)\end{array}$ & Open right hemicolectomy \\
\hline 5 & 54 & 1.4 & Adenocarcinoma & $\begin{array}{l}\| \mathrm{A} \\
(\mathrm{T} 3, \mathrm{NO}, \mathrm{MO})\end{array}$ & $\begin{array}{l}\text { Laparoscopic appendectomy with interval open right hemicolec- } \\
\text { tomy }\end{array}$ \\
\hline 6 & 49 & 1.3 & Adenocarcinoma & $\begin{array}{l}\text { IV } \\
(\mathrm{T} 3, \mathrm{~N} 2, \mathrm{M} 1)\end{array}$ & Open right hemicolectomy \\
\hline 7 & 23 & 1.3 & Neuroendocrine & $\begin{array}{l}\text { । } \\
(\mathrm{T} 1, \mathrm{NO}, \mathrm{MO})\end{array}$ & Laparoscopic appendectomy \\
\hline 8 & 71 & 1.2 & Neuroendocrine & $\begin{array}{l}\text { I } \\
(\mathrm{T} 1, \mathrm{NO}, \mathrm{MO})\end{array}$ & Open ileocecectomy with ileostomy \\
\hline 9 & 53 & 1.1 & Neuroendocrine & $\begin{array}{l}\text { । } \\
(\mathrm{T} 1, \mathrm{NO}, \mathrm{MO})\end{array}$ & Laparoscopic appendectomy \\
\hline 10 & 47 & 1.5 & Neuroendocrine & I $(\mathrm{T} 1, \mathrm{~N} 0, \mathrm{MO})$ & Laparoscopic appendectomy \\
\hline 11 & 47 & 0.7 & Neuroendocrine & $\begin{array}{l}\text { III } \\
(\mathrm{T} 4, \mathrm{NO}, \mathrm{MO})\end{array}$ & Laparoscopic appendectomy \\
\hline 12 & 46 & 1.1 & Neuroendocrine & I $(\mathrm{T} 1, \mathrm{NO}, \mathrm{MO})$ & Laparoscopic appendectomy \\
\hline 13 & 40 & 0.9 & Neuroendocrine & $\begin{array}{l}\text { I } \\
(\mathrm{T} 1, \mathrm{NO}, \mathrm{MO})\end{array}$ & Open appendectomy \\
\hline 14 & 28 & 1.0 & Neuroendocrine & $\begin{array}{l}\text { । } \\
(\mathrm{T} 1, \mathrm{NO}, \mathrm{MO})\end{array}$ & Open appendectomy \\
\hline 15 & 26 & 1.1 & Neuroendocrine & I & Open appendectomy \\
\hline 16 & 25 & 1.4 & Neuroendocrine & I $\mathrm{T} 1, \mathrm{NO}, \mathrm{MO})$ & Open appendectomy \\
\hline 17 & 23 & 1.1 & Neuroendocrine & I & Open appendectomy \\
\hline 18 & 10 & 1.1 & Neuroendocrine & I & Laparoscopic appendectomy \\
\hline 19 & 9 & 1.1 & Neuroendocrine & $\begin{array}{l}\text { I } \\
(\mathrm{T} 1, \mathrm{NO}, \mathrm{MO})\end{array}$ & Laparoscopic appendectomy \\
\hline 20 & 64 & 4.5 & LAMN & - & Laparoscopic appendectomy \\
\hline 21 & 58 & 1.5 & LAMN & - & Laparoscopic appendectomy \\
\hline 22 & 56 & 2.5 & LAMN & - & Laparoscopic appendectomy \\
\hline 23 & 55 & 1.5 & LAMN & - & Laparoscopic appendectomy \\
\hline 24 & 54 & 4.5 & LAMN & - & $\begin{array}{l}\text { Laparoscopic appendectomy converted to open right hemicolec- } \\
\text { tomy }\end{array}$ \\
\hline 25 & 50 & 1.2 & LAMN & - & $\begin{array}{l}\text { Open right hemicolectomy with resection of terminal ileum and } \\
\text { ileocolic anastomosis }\end{array}$ \\
\hline 26 & 47 & 5.5 & LAMN & - & Laparoscopic appendectomy \\
\hline 27 & 42 & 2.1 & LAMN & - & Open appendectomy \\
\hline
\end{tabular}

LAMN low-grade appendiceal mucinous neoplasm

and eight cases of low-grade appendiceal mucinous neoplasms (LAMN, $0.4 \%$ ). Twenty $(74.1 \%)$ of the patients were 40 years or older at the time of diagnosis. Moreover, the diameter of the appendix was greater or equal to $1.0 \mathrm{~cm}$ on pre-operative imaging in 25 of $27(92.6 \%)$ patients with appendiceal cancer. All patients with 
LAMN $(\mathrm{n}=8)$ had an appendiceal diameter greater than $1.0 \mathrm{~cm}$ with imaging findings consistent with mucocele.

A representation of patients divided by type of neoplasm and age is presented in Fig. 3. Adenocarcinoma only appeared in patients older than the age of 40 in the local cohort. Overall, older median age was associated with appendiceal adenocarcinoma: 65.0 IQR [52.8-75.3] vs. 47.0 IQR [25.5-54.5] years $(\mathrm{p}=0.004$; Table 1$)$.

\section{Discussion}

In this study we reviewed 2124 cases of acute appendicitis in two municipal hospitals. From our local experience, all cases of appendiceal adenocarcinoma were identified in patients older than 40 years, while cases of adenocarcinoma were reported in patients under 40 years in the NSQIP analysis. These data concur with a similar NSQIP analysis performed by Lu, et al. between 2016 and 2017 . Furthermore, these results were in agreement with those established in prior reports that support a significant association between increased risk of malignancy and increasing age in patients who have undergone surgical appendectomy for presumed appendicitis [17]. The rate of appendectomy at Jacobi Medical Center and North Central Bronx Hospital for acute appendicitis was 94\%, which also matched the United States NSQIP rate of 95\%. The rate of appendiceal cancer at Jacobi and North Central Bronx Hospital of 1.4\% was higher than the observed NSQIP rate of $0.53 \%$ for appendiceal cancer in acute appendicitis.
The incidence of appendiceal cancer rose from 2000 to 2009 in an analysis of the "Surveillance, Epidemiology, and End Results" (SEER) database [18]. In the setting of a progressively aging population, there is an observed increase in appendicitis in older adults. The management patterns of acute appendicitis are also evolving. Over the last several years, numerous studies have determined safety and efficacy of non-operative treatment with specific indications [1, 2, 7, 19-28]. Healthcare providers have been eager to adapt this new modality without consideration for possible long-term outcomes and oncologic ramification.

The COVID-19 pandemic has forced surgeons to alter their strategies in the management of acute appendicitis. Non-operative management, as well as the use of open appendectomy, reflect the poor guidelines available during the early stages of the SARS-CoV-2 pandemic [11]. This evolution has accelerated during the COVID-19 pandemic as non-operative management was encouraged by results from the CODA Trial [14] and American College of Surgeons guidelines [13].

The risk of appendiceal neoplasm increases with severe, complicated appendicitis [29]. Nevertheless, the severity of appendicitis may not be adequately determined by cross-sectional imaging [30]. CT failed to identify complicated disease in 187 of 837 patients (22\%) thought to have uncomplicated disease based on imaging characteristics alone. Nevertheless, in patients with few or no risk factors for perforation, surgery can be safely postponed

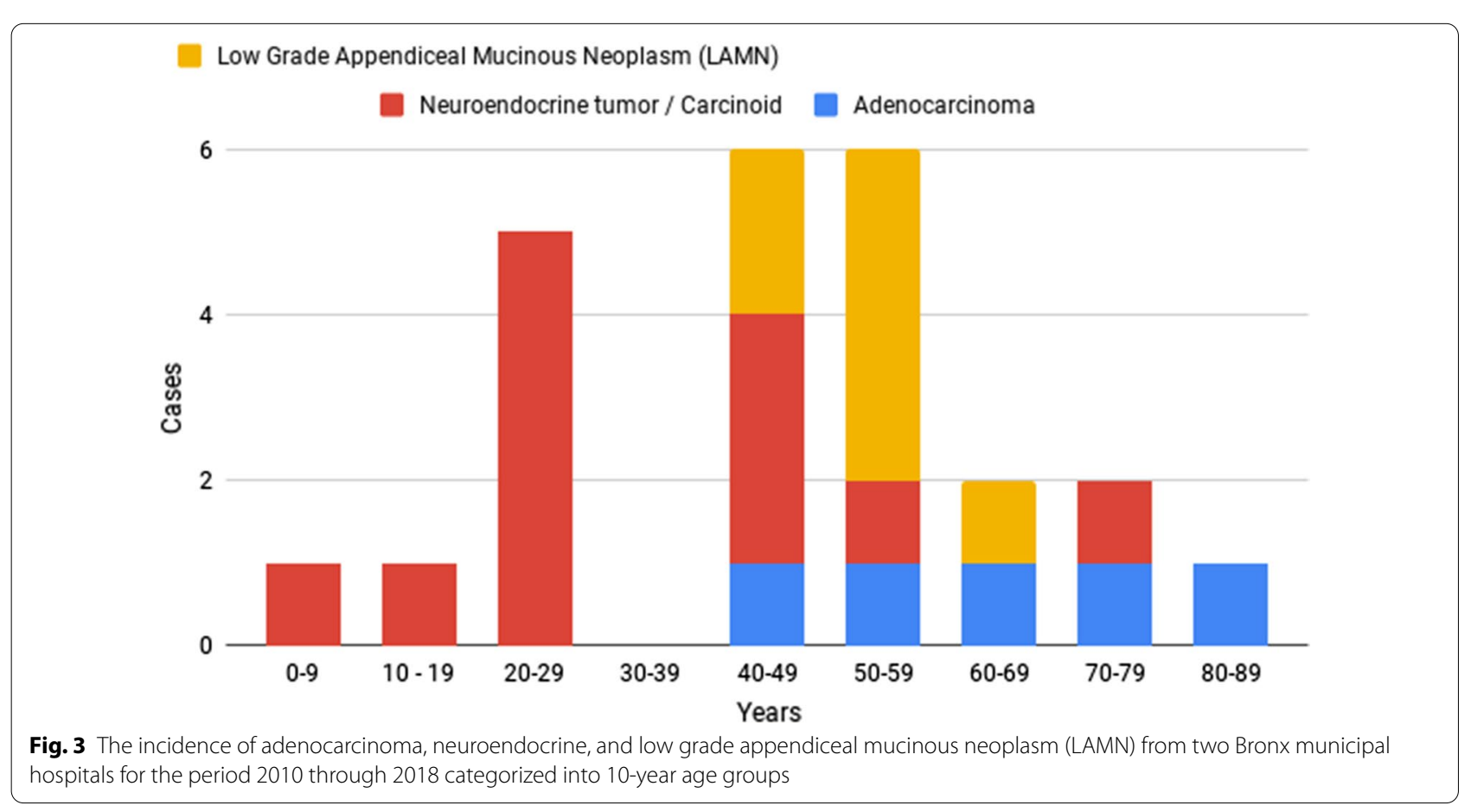


up to $7 \mathrm{~h}$. These findings may have real clinical consequences if patients with complicated disease are not recognized and are offered non-operative management.

To date, the only gold standard confirmatory test for appendiceal cancer is appendectomy that is usually performed for the presentation of acute appendicitis [31-33]. In a recent prospective study of acute complex appendicitis, patients with complicated appendicitis who underwent either surveillance or interval appendectomy were found to have an exceedingly high rate of malignancy that even resulted in premature discontinuation of a prospective study [17, 29, 34, 35]. Our findings corroborate recent findings in prospectively collected data, analyzed post-hoc, from the MUSTANG trial $[15,16]$. In our local study, 25 of 27 patients (93\%) had an appendiceal diameter greater than $1 \mathrm{~cm}$ (Table 2). Taken together we concur that age older than 40 and an appendiceal diameter greater than $1 \mathrm{~cm}$ on $\mathrm{CT}$ imaging must be considered risk factors for malignancy when considering appendectomy at initial presentation or at a later date $[15,16$, 36]. Patients with acute appendicitis managed with nonoperative treatment should be followed with screening colonoscopy and interval, full-dosed contrast enhanced CT imaging based on recommendations from the World Society of Emergency Surgery [37].

Analysis of the NSQIP data demonstrated that the incidence of appendiceal neoplasm was higher in older adults, with a marked increase after 50 years. Nonetheless, this incidence was lower than that observed in our urban, Bronx-based population (0.53\% vs. $1.4 \%)$. There are several explanations for this discrepancy. NSQIP does not capture every case of appendicitis. This leaves open the possibility that cases of appendiceal neoplasm were missed. This deficiency combined with the larger sample size may lead to a decrease in relative percentages of appendicitis in the NSQIP sample. Another point of possible discrepancy involved the pathologic diagnosis of appendiceal cancer. In the local group, anatomic pathology was performed and reviewed by one group. In NSQIP the data input was entered at the time of initial diagnosis based on a pathology report. It is possible that diagnostic criteria may vary from facility to facility across a national database. Regardless, the overall trends were similar. The incidence of appendiceal neoplasm, in particular appendiceal adenocarcinoma, increased with age and highlighted the need to consider the possibility of underlying malignancy in acute appendicitis in adults over the age of 40 .

Interestingly, our analysis of NSQIP data in patients with appendiceal cancer show that cancer, and in particular appendiceal adenocarcinoma can also occur in patients younger than 50 years of age. Our local data also suggest that the incidence of adenocarcinoma directly correlates with age. However, our local study was limited by its retrospective design and review of patient data from a now unmaintained, older electronic medical record. In the emergency surgery setting, patient followup was poor. Our municipal hospital system also serves underrepresented and an economically disadvantaged patient population with limited adherence in all surgical settings. Furthermore, the practice patterns at our institution, with inclusion of earlier data, reflected a surgical practice pattern that may be limited at other centers, especially outside the United States.

Appendiceal neoplasms are an orphan disease with a heterogeneous entity that escape straightforward classification or management [38-40]. The "screening test" for appendiceal cancer is appendectomy for patients who present emergently with acute appendicitis. Appendiceal cancer is rarely found by colonoscopy or as an incidental finding on CT scan or abdominal surgery for other reasons [41]. However, delayed diagnosis for appendiceal adenocarcinoma may have significant changes in staging and outcome. Further study of these tumors in the acute setting is required to adequately screen patients who may not be eligible for medical management or whose atypical presentation may highlight the need for further oncologic workup prior to surgical intervention.

\section{Conclusions}

Appendiceal neoplasms are a heterogeneous group of diseases that vary significantly in oncologic outcomes and are often diagnosed in the setting of acute appendicitis. Given the higher incidence of adenocarcinoma in older patients with appendicitis, patients over 40 years of age should be counseled regarding the risk of malignancy when considering non-operative therapy.

\section{Acknowledgements \\ We thank the Physician Affiliate Group of New York (PAGNY) Health and Research Foundation who supported this project and is committed to enhancing health on all of New York City's diverse communities. We also thank Sherrie R. White, MD and Joanne Stern, who aided in data collection. \\ Authors' contributions \\ VSA and JPS are both co-first authors of this manuscript and contributed equally in the collection, categorization, organization, and analysis of the data and wrote the manuscript. VA contributed to the writing of the manuscript and data collection. DR conceived the design and collection of the data. JM obtained the National Surgery Quality Improvement Program (NSQIP) data and helped with the manuscript preparation. PKK conceived the original idea, supervised manuscript preparation, and took the lead of the project. All authors reviewed the manuscript. All authors read and approved the final manuscript.}

\section{Funding}

Not applicable.

\section{Availability of data and materials}

De-identified patient data are available for external review upon reasonable request. Requests for identified patient data will be submitted to the Internal Review Board of Albert Einstein College of Medicine for consideration. 


\section{Declarations}

Ethics approval and consent to participate

This study received ethics approval by the Albert Einstein College of Medicine Internal Review Board (IRB) prior to data collection and analysis. Consent to participate was waived by the IRB.

\section{Consent for publication}

All authors approved this manuscript and consent for its publication.

\section{Competing interests}

The authors declare no competing interests.

\section{Author details}

${ }^{1}$ Jacobi Medical Center, Bronx, NY, USA. ${ }^{2}$ North Central Bronx Hospital, Bronx, NY, USA. ${ }^{3}$ Montefiore Medical Center, Bronx, NY, USA. ${ }^{4}$ Albert Einstein College of Medicine, Bronx, NY, USA. ${ }^{5}$ Jacobi Medical Center, 1400 Pelham Parkway, Building 1, Room 510, Bronx, NY 10461, USA.

Received: 8 February 2021 Accepted: 21 April 2021 Published online: 02 May 2021

\section{References}

1. Ferris M, Quan S, Kaplan BS, Molodecky N, Ball CG, Chernoff GW, et al. The global incidence of appendicitis: a systematic review of populationbased studies. Ann Surg. 2017;266(2):237-41.

2. Mason RJ. Surgery for appendicitis: is it necessary? Surg Infect (Larchmt) 2008;9(4):481-8.

3. Podda M, Gerardi C, Cillara N, Fearnhead N, Gomes CA, Birindelli A, et al. Antibiotic treatment and appendectomy for uncomplicated acute appendicitis in adults and children: a systematic review and meta-analysis. Ann Surg. 2019;270(6):1028-40.

4. Di Saverio S, Sibilio A, Giorgini E, Biscardi A, Villani S, Coccolini F, et al. The NOTA Study (Non Operative Treatment for Acute Appendicitis): prospective study on the efficacy and safety of antibiotics (amoxicillin and clavulanic acid) for treating patients with right lower quadrant abdominal pain and long-term follow-up of conservatively treated suspected appendicitis. Ann Surg. 2014;260(1):109-17.

5. Kadera SP, Mower WR, Krishnadasan A, Talan DA. Patient perspectives on antibiotics for appendicitis at one hospital. J Surg Res. 2016;201(2):253-7.

6. Loftus TJ, Raymond SL, Sarosi GA Jr, Croft CA, Smith RS, Efron PA, et al. Predicting appendiceal tumors among patients with appendicitis. J Trauma Acute Care Surg. 2017:82(4):771-5.

7. McCutcheon BA, Chang DC, Marcus LP, Inui T, Noorbakhsh A, Schallhorn $C$, et al. Long-term outcomes of patients with nonsurgically managed uncomplicated appendicitis. J Am Coll Surg. 2014;218(5):905-13.

8. Kessler U, Mosbahi S, Walker B, Hau EM, Cotton M, Peiry B, et al. Conservative treatment versus surgery for uncomplicated appendicitis in children: a systematic review and meta-analysis. Arch Dis Child. 2017:102(12):1118-24.

9. Huang L, Yin Y, Yang L, Wang C, Li Y, Zhou Z. Comparison of antibiotic therapy and appendectomy for acute uncomplicated appendicitis in children: a meta-analysis. JAMA Pediatr. 2017;171(5):426-34.

10. CODA Collaborative. A randomized trial comparing antibiotics with appendectomy for appendicitis. N Engl J Med. 2020;383(20):1907-19.

11. lelpo B, Podda M, Pellino G, Pata F, Caruso R, Gravante G, et al. Global attitudes in the management of acute appendicitis during COVID-19 pandemic: ACIE apply study. Br J Surg. 2020. https://doi.org/10.1002/bjs. 11999.

12. Patel VK, Ye K, In H, Scheinfeld MH. Non-operative management for acute appendicitis during the COVID-19 pandemic does not increase the rate of complications. J Gastrointest Surg. 2020. https://doi.org/10.1007/ s1 1605-020-04844-8.

13. https://www.facs.org/covid-19/clinical-guidance/elective-case/emerg ency-surgery. Accessed 1 Feb 2021.

14. Davidson GH, Flum DR, Talan DA, Kessler LG, Lavallee DC, Bizzell BJ, et al. Comparison of outcomes of antibiotic drugs and appendectomy (CODA) trial: a protocol for the pragmatic randomised study of appendicitis treatment. BMJ Open. 2017;7(11):e016117.

15. Yeh DD, Eid Al, Young KA, Wild J, Kaafarani HMA, Ray-Zack M, et al. Multicenter study of the treatment of appendicitis in america: acute, perforated, and gangrenous (MUSTANG), an EAST multicenter study. Ann Surg. 2021;273(3):548-56.

16. Yeh DDEA, Young KA, Wild J, Kaafarani HMA, Ray-Zack M, et al. Multicenter study of the treatment of appendicitis in america: acute, perforated, and gangrenous (MUSTANG), an EAST multicenter study. Ann Surg. 2019;273:548-56.

17. Lu P, McCarty JC, Fields AC, Lee KC, Lipsitz SR, Goldberg JE, et al. Risk of appendiceal cancer in patients undergoing appendectomy for appendicitis in the era of increasing nonoperative management. J Surg Oncol. 2019:120(3):452-9.

18. Marmor S, Portschy PR, Tuttle TM, Virnig BA. The rise in appendiceal cancer incidence: 2000-2009. J Gastrointest Surg. 2015;19(4):743-50.

19. Styrud J, Eriksson S, Nilsson I, Ahlberg G, Haapaniemi S, Neovius G, et al. Appendectomy versus antibiotic treatment in acute appendicitis: a prospective multicenter randomized controlled trial. World J Surg. 2006;30(6):1033-7.

20. Vons C, Barry C, Maitre S, Pautrat K, Leconte M, Costaglioli B, et al. Amoxicillin plus clavulanic acid versus appendicectomy for treatment of acute uncomplicated appendicitis: an open-label, non-inferiority, randomised controlled trial. Lancet. 2011;377(9777):1573-9.

21. Teixeira PG, Demetriades D. Appendicitis: changing perspectives. Adv Surg. 2013:47:119-40.

22. Sippola S, Gronroos J, Tuominen R, Paajanen H, Rautio T, Nordstrom P, et al. Economic evaluation of antibiotic therapy versus appendicectomy for the treatment of uncomplicated acute appendicitis from the APPAC randomized clinical trial. Br J Surg. 2017;104(10):1355-61.

23. Sippola S, Grönroos J, Sallinen V, Rautio T, Nordström P, Rantanen T, et al. A randomised placebo-controlled double-blind multicentre trial comparing antibiotic therapy with placebo in the treatment of uncomplicated acute appendicitis: APPAC III trial study protocol. BMJ Open. 2018;8(11):e023623.

24. Simillis C, Symeonides P, Shorthouse AJ, Tekkis PP. A meta-analysis comparing conservative treatment versus acute appendectomy for complicated appendicitis (abscess or phlegmon). Surgery. 2010;147(6):818-29.

25. Salminen P, Tuominen R, Paajanen H, Rautio T, Nordström P, Aarnio $M$, et al. Five-year follow-up of antibiotic therapy for uncomplicated acute appendicitis in the APPAC randomized clinical trial. JAMA. 2018;320(12):1259-65.

26. Salminen P, Paajanen H, Rautio T, Nordstrom P, Aarnio M, Rantanen T, et al. Antibiotic therapy vs appendectomy for treatment of uncomplicated acute appendicitis: the APPAC randomized clinical trial. JAMA. 2015;313(23):2340-8.

27. Haijanen J, Sippola S, Grönroos J, Rautio T, Nordström P, Rantanen T, et al. Optimising the antibiotic treatment of uncomplicated acute appendicitis: a protocol for a multicentre randomised clinical trial (APPAC II trial). BMC Surg. 2018;18(1):117.

28. Prechal D, Damirov F, Grilli M, Ronellenfitsch U. Antibiotic therapy for acute uncomplicated appendicitis: a systematic review and meta-analysis. Int J Colorectal Dis. 2019:34(6):963-71.

29. Lietzen E, Gronroos JM, Mecklin JP, Leppaniemi A, Nordstrom P, Rautio T, et al. Appendiceal neoplasm risk associated with complicated acute appendicitis-a population based study. Int J Colorectal Dis. 2019:34(1):39-46.

30. Lastunen K, Leppäniemi A, Mentula P. Perforation rate after a diagnosis of uncomplicated appendicitis on CT. BJS Open. 2021;5(1):zraa034.

31. Talan DA. Cancer of the appendix and nonoperative treatment of appendicitis shared decision making. J Surg Oncol. 2019;120(6):1060-1.

32. Seawell J, Sciarretta JD, Pahlkotter M, Muertos K, Onayemi A, Davis JM The understated malignancy potential of nonoperative acute appendicitis. Am Surg. 2019:85(7):712-6.

33. Mällinen J, Rautio T, Grönroos J, Rantanen T, Nordström P, Savolainen $\mathrm{H}$, et al. Risk of appendiceal neoplasm in periappendicular abscess in patients treated with interval appendectomy vs follow-up with magnetic resonance imaging: 1-year outcomes of the peri-appendicitis acuta randomized clinical trial. JAMA Surg. 2019;154(3):200-7.

34. Mallinen J, Rautio T, Gronroos J, Rantanen T, Nordstrom P, Savolainen $\mathrm{H}$, et al. Risk of appendiceal neoplasm in periappendicular abscess in 
patients treated with interval appendectomy vs follow-up with magnetic resonance imaging: 1-year outcomes of the peri-appendicitis acuta randomized clinical trial. JAMA Surg. 2019;154(3):200-7.

35. Limaiem F, Arfa N, Marsaoui L, Bouraoui S, Lahmar A, Mzabi S. Unexpected histopathological findings in appendectomy specimens: a retrospective study of 1627 cases. Indian J Surg. 2015;77(Suppl 3):1285-90.

36. Naar L, Kim P, Byerly S, Vasileiou G, Zhang H, Yeh DD, et al. Increased risk of malignancy for patients older than 40 years with appendicitis and an appendix wider than $10 \mathrm{~mm}$ on computed tomography scan: a post hoc analysis of an EAST multicenter study. Surgery. 2020. https://doi.org/10. 1016/j.surg.2020.05.044

37. Di Saverio S, Podda M, De Simone B, Ceresoli M, Augustin G, Gori A, et al. Diagnosis and treatment of acute appendicitis: 2020 update of the WSES Jerusalem guidelines. World J Emerg Surg. 2020;15(1):27.

38. Wright GP, Mater ME, Carroll JT, Choy JS, Chung MH. Is there truly an oncologic indication for interval appendectomy? Am J Surg. 2015;209(3):442-6.
39. West NE, Wise PE, Herline AJ, Muldoon RL, Chopp WV, Schwartz DA. Carcinoid tumors are 15 times more common in patients with Crohn's disease. Inflamm Bowel Dis. 2007;13(9):1129-34.

40. Todd RD, Sarosi GA, Nwariaku F, Anthony T. Incidence and predictors of appendiceal tumors in elderly males presenting with signs and symptoms of acute appendicitis. Am J Surg. 2004;188(5):500-4.

41. Subramanya D, Grivas PD, Styler M. Appendiceal carcinoma: a diagnostic and therapeutic challenge. Postgrad Med. 2008;120(4):95-100.

\section{Publisher's Note}

Springer Nature remains neutral with regard to jurisdictional claims in published maps and institutional affiliations.
Ready to submit your research? Choose BMC and benefit from:

- fast, convenient online submission

- thorough peer review by experienced researchers in your field

- rapid publication on acceptance

- support for research data, including large and complex data types

- gold Open Access which fosters wider collaboration and increased citations

- maximum visibility for your research: over $100 \mathrm{M}$ website views per year

At BMC, research is always in progress.

Learn more biomedcentral.com/submissions 